\title{
The Method of dealing with Geomagnetic Anomaly caused by Movable Magnetic Objects in Drawing the Geomagnetic Chart
}

\author{
Wang Ming ${ }^{1}$, Kang Chong ${ }^{2}$, Wan Shengwei ${ }^{2}$, Fan Liming ${ }^{3}$ and Zhang Xiaojun ${ }^{2}$ \\ ${ }^{1}$ College of Mechanical and Electrical Engineering, Harbin Engineering University, \\ Harbin 150001 P. R. China \\ ${ }^{2}$ Science College, Harbin Engineering University, Harbin 150001 P. R. China \\ ${ }^{3}$ Genersoft Company of Inspur Group, Jinan 250101 P. R. China
}

\begin{abstract}
The local geomagnetic chart is an effective tool to describe the geomagnetic information in the region accurately and visually which is a reference in application for future. Precision of local geomagnetic chart directly affects its value. When the movable magnetic objects are in the region, the distribution of geomagnetic field will be changed. When the movable magnetic objects are out the region, the distribution of geomagnetic field will recover to normal situation. Thus, magnetic objects can bring the error to some measured data. As an result, precision of the geomagnetic chart will reduce. On the basis of analysis of the existing geomagnetic model, the method to eliminate the movable geomagnetic anomaly in drawing the chart is proposed which is based on Taylor polynomial model. In this method, affected regions by magnetic object are gotten, the geomagnetic model are built in these affected region by precise data, the measured data in these region is replayed with model data, finally, the geomagnetic chart is draw by combined data using Kriging interpolation algorithm. The experiment results show that the method can improve the precision of the local magnetic chart when the movable magnetic objects exist in the measurement region.
\end{abstract}

Keywords: Geomagnetic anomaly, Geomagnetic field, Geomagnetic normal field, Taylor polynomial, Least squared estimation

\section{Introduction}

Geomagnetic field is the basic physical field of the Earth system and inherent resources of the earth, which can be used as the natural coordinate system for aviation, aerospace, marine and can be used in positioning and orientation and attitude control of spacecraft or vessels. The magnetic navigation technology belongs to the passive autonomous navigation that is simple, efficient, reliable performance, strong anti-interference though the spatial distribution of the Earth's magnetic field. Geomagnetic maps are a powerful tool that can made geomagnetic information expressed with accurately, detailed, visually. Therefore, based on the geomagnetic chart, carry out the corresponding geomagnetic navigation and its applications can be carried out.

High-precision and high-density magnetic survey is the basis for the preparation of high-precision geomagnetic map [1-3]. Typically there are three ways to get geomagnetic data: namely geomagnetic field data or measurement points, aircraft aerial magnetic survey data and satellite data [4-5]. The local 
geomagnetic map can be improved through tow methods: (1) Increase the data density of the geomagnetic field in the measuring region: through the method of data fitting or interpolation, we can improve the geomagnetic data density, getting geomagnetic map with certain accuracy. Fitting interpolation methods commonly used are: Distance weighted interpolation, nearest neighbor interpolation method, the minimum curvature method, trend surface fitting, Kriging interpolation method, etc. (2) Improve the accuracy of geomagnetic field data in the measuring region: using geomagnetic measuring equipment with high precision, removing the region of the movable magnetic body, deleting the magnetic filter geomagnetism data polluted by the movable magnetic body, etc.

Geomagnetic field model is a method to draw large-scale regional geomagnetic normal field, such as An Zhenchang used the spherical cap harmonic model to draw the map of Chinese regional geomagnetic field [7] and the map of East Asia magnetic field [8-9]; Chen P C used the Taylor polynomial to construct Chongqing Beibei geomagnetic field model [10];Gu Zuowen used the geomagnetic observation data of the Beijing Tianjin Hebei region and the spherical cap harmonic model to draw the map of the regional geomagnetic field [11].

When measuring the geomagnetic field in a small area, there may be movable magnetic object in the measuring area (such as cars, ships, etc.) The geomagnetic field distribution would be changed in the surrounding space when magnetic objects were in measuring region. The magnetic field distribution recovered to its original state when the magnetic objects were left. When the movable magnetic objects exist in the measuring region, the accuracy of measurement data will be reduced. When removed the affected data, the density of geomagnetic field data in the region will be lower. These cases both can affect the accuracy of the magnetic map. In this paper, in order to improve the accuracy of geomagnetic field map, the method is used to eliminate the influence of the magnetic anomalies of el the movable magnetic objects on geomagnetic mapping which is to build the geomagnetic model in the affected region.

\section{Kriging Interpolation Algorithm}

The is no an absolute best algorithm in the spatial interpolation algorithm. The suitable interpolation algorithm is chosed based on actual needs. The attribute between geomagnetic field and terrain is relatively same, as there is a correlation between the spatial attributes. Since kriging interpolation algorithm has a high degree of approximation of the performance space in the property-related issues. Therefore, the Kriging interpolation method is used in this paper.

Kriging interpolation method is the method of the linear unbiased optimal estimation to the unknown sample points, which is named after the French scientist Krige. The basic process that Spatial interpolation with the Kriging interpolation method can be divided into: (1) Obtain the experimental variogram function by measured data. (2) Obtain the theory variogram function by fitted the experimental variogram function. (3) Carry out spatial interpolation on no sample points. The Kriging interpolation method not only be considered the 
distance of points, but be considered the spatial distribution relationship between known sample points and unknown sample points. Kriging interpolation method not only can reflect the structural components of spatial data and the random distribution of the variables, but also describe the error message, thus it has a wide range of application.

Common variogram function model includes constant model, exponential model, the spherical model, Gaussian model, etc. In order to optimize model, trend, residuals, covariates, covariance and other factors are introduced. Thus, many kind of Kriging interpolation method have evolved. In this paper, the ordinary Kriging interpolation method is analyzed, its principle is as follows:

The position of the sample point is $x_{1}$ in the region and the measured value of the point is $M\left(x_{i}\right), i=1,2, \cdots, n$. Then estimated value $M^{*}\left(x_{0}\right)$ in the prediction point $\mathrm{x}_{0}$ can be expressed by Linear Unbiased Estimation of above-mentioned sample points, as follows:

$$
\begin{gathered}
\left\{\begin{array}{l}
M^{*}\left(x_{0}\right)=\sum_{i=1}^{n} \lambda_{i} M\left(x_{i}\right) \\
\sum_{i=1}^{n} \lambda_{i}=1
\end{array}\right. \\
E\left\{\left[M\left(x_{0}\right)-M^{*}\left(x_{0}\right)\right]^{2}\right\}=E\left\{\left[M\left(x_{0}\right)-\sum_{i=1}^{n} \lambda_{i} M\left(x_{i}\right)\right]^{2}\right\}
\end{gathered}
$$

Where ${ }^{\lambda_{i}}$ are the weight coefficients of measured points.

In order to get the coefficients $\lambda_{i}$, the variogram function is introduced, which is defined as:

$$
\gamma\left(x_{i}, x_{j}\right)=\gamma\left(x_{i}-x_{j}\right)=\frac{1}{2} E\left[\mathrm{Z}\left(x_{i}\right)-\mathrm{Z}\left(x_{j}\right)\right]^{2}
$$

The formula (1) can be transformed into:

$$
\left\{\begin{array}{l}
\sum_{i=1}^{n} \lambda_{i} \gamma\left(x_{i}-x_{j}\right)+\mu=\gamma\left(x_{i}-x_{0}\right), j=1,2 \cdots n \\
\sum_{i=1}^{n} \lambda_{i}=1
\end{array}\right.
$$

Order that the matrix $K, \lambda$ and $D$ are expressed as follown:

$$
K=\left[\begin{array}{cccc}
\gamma\left(x_{1}-x_{1}\right) & \gamma\left(x_{2}-x_{1}\right) & \cdots & \gamma\left(x_{n}-x_{1}\right) \\
\gamma\left(x_{1}-x_{2}\right) & \gamma\left(x_{2}-x_{2}\right) & \cdots & \gamma\left(x_{n}-x_{2}\right) \\
\vdots & \vdots & \vdots & \vdots \\
\gamma\left(x_{1}-x_{n}\right) & \gamma\left(x_{2}-x_{n}\right) & \cdots & \gamma\left(x_{n}-x_{n}\right)
\end{array}\right] \quad \lambda=\left[\begin{array}{c}
\lambda_{1} \\
\lambda_{2} \\
\vdots \\
\lambda_{n}
\end{array}\right] \quad D=\left[\begin{array}{c}
\gamma\left(x_{1}-x_{0}\right) \\
\gamma\left(x_{2}-x_{0}\right) \\
\vdots \\
\gamma\left(x_{n}-x_{0}\right)
\end{array}\right]
$$


Therefore, the matrix in the formula (1) can be expressed as

$$
K \lambda=D
$$

As long as we get variogram function $\gamma$, we can calculate value a by the formula(1), and then we can obtain the interpolated value in the space position by the formula (6)

Sample variance function:

Assuming $M\left(x_{1}\right), M\left(x_{2}\right), \cdots M\left(x_{n}\right)$ is a sample of the data, under the inherent assumptions :

$$
E[M(x+h)-M(x)]=0, \quad E\left\{[M(x+h)-M(x)]^{2}\right\}=2 \gamma(h)
$$

Sample variogram function value can be calculated by the formula (7)

$$
\gamma^{*}(h)=\frac{1}{2 N_{h}} \sum_{i=1}^{n}\left[z\left(x_{i}+h\right)-z\left(x_{i}\right)\right]^{2}
$$

where $h$ is the distance, $N_{h}$ is the number of samples between $\left(x_{i}+h, x_{i}\right)$

\section{The Research on High Precise of Regional Geomagnetic Model}

Taylor polynomials can make good use of exponential basis functions to characterize changes in the geomagnetic field and it is a purely mathematical approach so that it has good spatial resolution. Therefore, Taylor polynomial can be used in small-scale region.

The geomagnetic model of Taylor polynomials [12] is followed as:

$$
F(\varphi, \lambda)=\sum_{n=0}^{N} \sum_{m=0}^{n} A_{n m}(\Delta \varphi)^{n-m}(\Delta \lambda)^{m}
$$

Where, $F$ is the geomagnetic field value of spatial location, $\Delta \varphi=\varphi-\varphi_{0}$, $\Delta \lambda=\lambda-\lambda_{0}, \varphi$ is the longitude, $\lambda$ is the latitude, $\varphi_{0}$ is the longitude of origin point, $\lambda_{0}$ is he latitude of origin point, $N$ is the cutoff order of the Taylor polynomial.

There is very small changes in latitude and longitude in small-scale region, which is lead to large error. However, It is easy to implement grid measurement in this region. Therefore, $\varphi$ is took as coordinate points in the $\mathrm{x}$ direction and $\lambda$ is took as coordinate points in the $\mathrm{y}$ direction. In this press, $\Delta \varphi, \Delta \lambda$ can be expressed as: 


$$
\left\{\begin{array}{l}
\Delta \varphi=\left[\varphi-\frac{1}{2}\left(\varphi_{\max }+\varphi_{\min }\right)\right] /\left[\left(\varphi_{\max }-\varphi_{\min }\right)\right] \\
\Delta \lambda=\left[\lambda-\frac{1}{2}\left(\lambda_{\max }+\lambda_{\min }\right)\right] /\left[\left(\lambda_{\max }-\lambda_{\min }\right)\right]
\end{array}\right.
$$

Where, $\varphi_{\max }$ is the maximum coordinate of $\mathrm{x}$ direction, $\varphi_{\min }$ is the minimum coordinate of $\mathrm{x}$ direction, $\lambda_{\max }$ is the maximum coordinate of $\mathrm{x}$ direction, $\lambda_{\min }$ is the minimum coordinate of $\mathrm{x}$ direction.

It has been proved that this change only affect the relative size of the model coefficients $A_{n m}$, and almost no effect for $F$.

The coefficients $A_{n m}$ generally obtained using the least squares method. The principle of the least squares method is followed:

$\rho=\sum_{k=1}^{K}\left[f_{k}\left(\varphi_{k}, \lambda_{k}\right)-F_{N}\left(\varphi_{k}, \lambda_{k}\right)\right]^{2}=\sum_{k=1}^{K}\left[f_{k}\left(\varphi_{k}, \lambda_{k}\right)-\sum_{n=0}^{N} \sum_{m=0}^{n} A_{n m}\left(\Delta \varphi_{k}\right)^{n-m}\left(\Delta \lambda_{k}\right)^{m}\right]^{2}=\min$

There, $f_{k}(\varphi, \lambda)$ is the measured geomagnetic filed value of $\left(\varphi_{k}, \lambda_{k}\right)$ point, $F_{N}\left(\varphi_{k}, \lambda_{k}\right)$ is the model value of $\left(\varphi_{k}, \lambda_{k}\right)$ point, $\mathrm{K}$ is total number of measured points, required $K>(N+1)(N+2) / 2$.

It can been seen that $\rho$ is the extremum problem of multi-function $A_{i j}$. Some results are gotten using the necessary conditions of multi-function:

$$
\frac{\Delta \rho}{\partial A_{i j}}=2\left\{\sum_{k=1}^{K}\left[f_{k}\left(\varphi_{k}, \lambda_{k}\right)-F_{N}\left(\varphi_{k}, \lambda_{k}\right)\right]\right\}^{*}\left(\Delta \varphi_{k}\right)^{i-j}\left(\Delta \lambda_{k}\right)^{j}=0
$$

By simplifying the formula:

$$
\sum_{n=0}^{N} \sum_{m=0}^{n} A_{n m} \sum_{k=1}^{K}\left(\Delta \varphi_{k}\right)^{n-m+i-j}\left(\Delta \lambda_{k}\right)^{m+j}=\sum_{k=1}^{K} f_{k}\left(\varphi_{k}, \lambda_{k}\right)\left(\Delta \varphi_{k}\right)^{i-j}\left(\Delta \lambda_{k}\right)^{j}
$$

The model coefficients $A_{n m}$ can be calculated though the Equations (11).

There are two main points needed to been considered when Taylor polynomial model is build. (1) The boundary effects of model: As measured data points are less on the region boundary, and the points are uneven distribution, which causes the model result is large different with actual situation at the border. That is called boundary effect. (2) The cutoff order of Taylor polynomials model: Theoretically, the greater the cutoff order, the higher the accuracy of model. But It is not exactly consistent with actual situation because the uneven distribution of the measured points, or less the measured points at the region boundary in the geomagnetic measurement lead to the boundary effect more and more serious, as the cutoff order increase. 
Therefore, in order to improve accuracy of the model and to avoid boundary effects, it is necessary to improve as much as possible at the boundary data density of measurement points. The number of measured points is easy to increase at the boundary and the even distribution of measured points is easy to release in small-scale region. Meanwhile, data interpolation can be used to improve the density of data points in the boundary. The steps of avoiding boundary effects of Taylor polynomial is as follows:

(1) Measure geomagnetic data throughout the region, and draw the regional geomagnetic contour;

(2) Determine the magnetic objects affected area by searching the geomagnetic data on the contour;

(3) Improve the data density of boundary points using the interpolation algorithm on the unaffected data at the boundary;

(4) Calculate the model coefficients $A_{n m}$ using the interpolated data.

The interpolated points at the boundary are added, evenly distributed data at the boundary are restricted the boundary effects, and the cutoff order of Taylor polynomial model is increased. After over processing, the boundary effects of model will be improved. Therefore, the accuracy of geomagnetic normal field in region will be improved.

\section{Elimination Method}

In order to improve the accuracy of the model region and avoid boundary effects, the data density of points at the region boundary should be increased as much as possible. When the measurement in the small-scale region, it is very easy to increase the number of measurement points and achieve the local distribution of measuring points at the boundary region. Meanwhile, doing interpolate for boundary measured data can further increase the density of data points in the boundary. In order to avoid boundary effects of Taylor polynomials, the following specific implementation methods should be taken.

(1) Obtain the value of the geomagnetic field at the sample points in the measurement region by actual measurement.

(2) Gridded processing of measured data by Kriging interpolation method.

(3) By analytic signal $A=\sqrt{(\nabla x)^{2}+(\nabla y)^{2}}$, determine the center position of the magnetic anomaly. Based on the value of the reference point geomagnetic field value, determine the scope of the magnetic anomalies.

(4) Around the outside of the sphere of influence, uniformly select a certain number of geomagnetic data, to strike geomagnetic model in the sphere of influence. 
(5) Replay the corresponding measured data with the theoretical normal geomagnetic field data in the affected region which are calculated by the geomagnetic model.

(6) Use the replaced geomagnetic data to draw local geomagnetic map

\section{Experiment Results and Analysis}

Measurement region is $18 \times 18$ uniform grids, pitch and line space is $0.5 \mathrm{~m}$. The magnetic object (the robot: length-1.5m, width- $0.6 \mathrm{~m}$, height $-0.8 \mathrm{~m}$ ) was placed in the centre of this region. Since the basic structure of the robot is constituted by a ferromagnetic material, the magnetic field around the object was changed because of the magnetization of object. The geomagnetic field value of this region was measured by grid measurements. Then the contour of local geomagnetic field was draw, as shown in Figure 1.

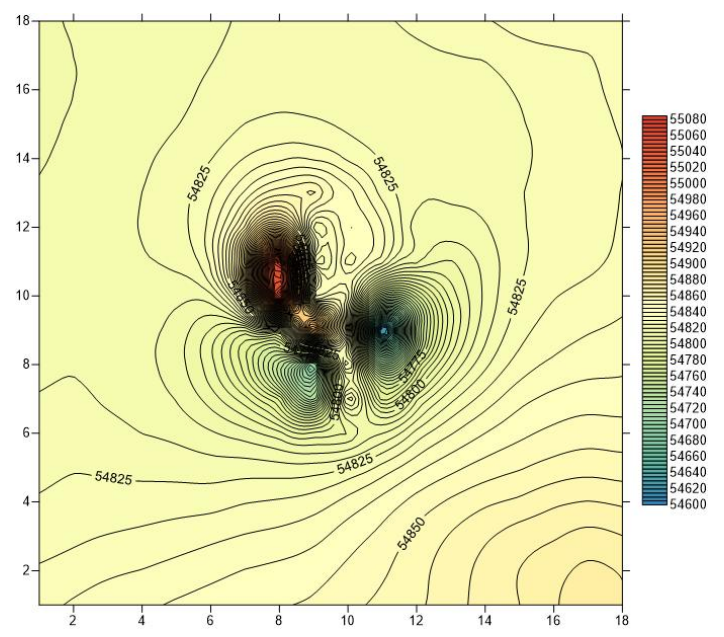

Figure 1. The Local Geomagnetic Chart with Robot

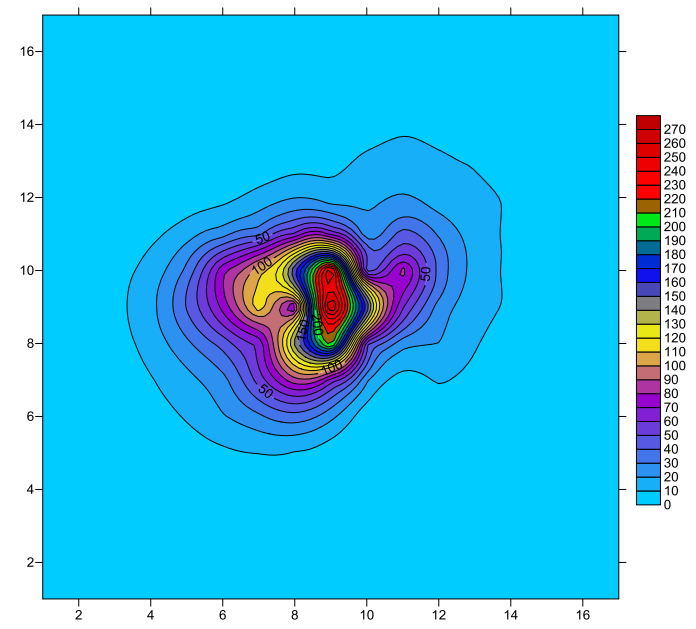

Figure 2. The Chart of Analytic Signal 
Generally, analytic signal maxima of amplitude correspond to the location of the field source. Its abnormal morphology could not be affected by magnetization direction substantially and having a characteristic with derivative anomaly. It has a more sensitive response to the boundary of the field source, and have different display to the shape and size of the source bodies. Analytic signal diagram in the region in Figure 2.

In Figure 2, it can be seen that the maximum amplitude of analytic signal is in the red area, magnetic anomaly center position should be located within the red area. In order to calculate more convenient, the position of magnetic object center was selected at [9, 9]. The difference value between two adjacent points whose distance was 0.5 meter was $4 \mathrm{nT}$. The range of magnetic anomalies in the affected region was found by data search: The horizontal coordinate was from 5 to 13 and the vertical coordinate was from 6 to 16. Therefore, the geomagnetic field at the location of the boundary region is not affected largely. The density of data at the boundary region was increased by kriging interpolation algorithm. The geomagnetic field model was build though the interpolated data and not affected data. It will not only ensure the accuracy of the normal geomagnetic field data, but also avoid boundary effects.

The cutoff order $\mathrm{N}$ of Taylor polynomial model is selected from 3 to 8 , and the root mean square error (RMSE) corresponding to $\mathrm{N}$ were calculated, the result is shown in Figure 2.

Selecting the cutoff order of Taylor polynomial $N: 3 \sim 8$ and calculate a corresponding root-mean-square error respectively. As shown in Table 1.

Table 1. The Distribution of Root Mean Square Error Corresponding to Cutoff Order N

\begin{tabular}{c|rccccc}
\hline Cutoff Order & 3 & 4 & 5 & 6 & 7 & 8 \\
\hline RMSE & $\begin{array}{r}7 . \\
45\end{array}$ & 7.63 & 7.87 & 7.81 & 7.94 & 172.56 \\
\hline
\end{tabular}

It can be seen from Table 1 that the root mean square error change little when cutoff order $\mathrm{N}$ is changed from 3 to 7; the root mean square error change rapidly when $\mathrm{N}=8$. In other word, boundary effects of this model has become very apparent when $\mathrm{N}=8$. Thus, $\mathrm{N}=3 \sim 7$ is considered in this model. In order to calculate simply and rapidly, $\mathrm{N}=3$ is selected in this model. The Parameters are shown in Table 1.

Table 2. Model Coefficients of Taylor Polynomial

\begin{tabular}{cccc}
\hline \multicolumn{4}{c}{$A_{\mathrm{nm}}(N=3)$} \\
\hline$A_{00}$ & 54855.82 & $A_{22}$ & 0.1334717 \\
$A_{10}$ & 2.964645 & $A_{30}$ & -0.0121762 \\
$A_{11}$ & -1.458558 & $A_{31}$ & -0.0038934 \\
$A_{20}$ & 0.0167891 & $A_{32}$ & 0.0075271 \\
$A_{21}$ & -0.0574168 & $A_{33}$ & 0.0017592 \\
\hline
\end{tabular}


The local geomagnetic field contours is draw using the model whose cutoff order $\mathrm{N}$ equals 3 . The contour is shown in Figure 4.

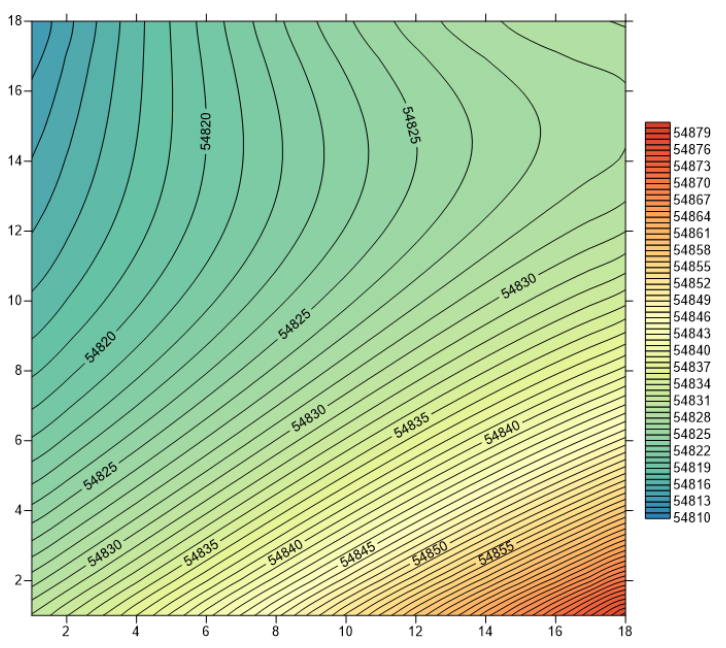

Figure 3. The Local Geomagnetic Contour Chart Under the Model of $\mathrm{N}=3$

In the scope of influence of magnetic anomalies, the affected measured data was replayed with theoretical data under the geomagnetic model. The local geomagnetic chart was draw using the new data. As shown in Figure 5.

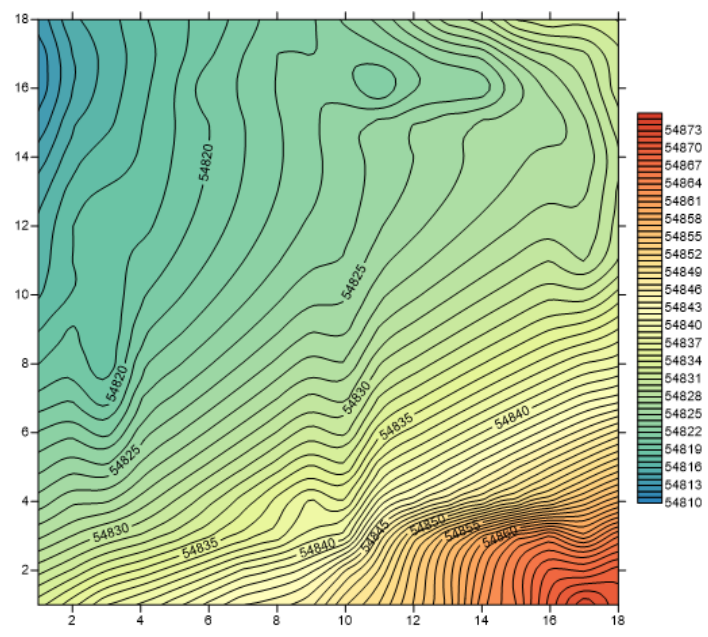

Figure 4. The Local Geomagnetic Contour Chart after Eliminating Magnetic Anomaly 


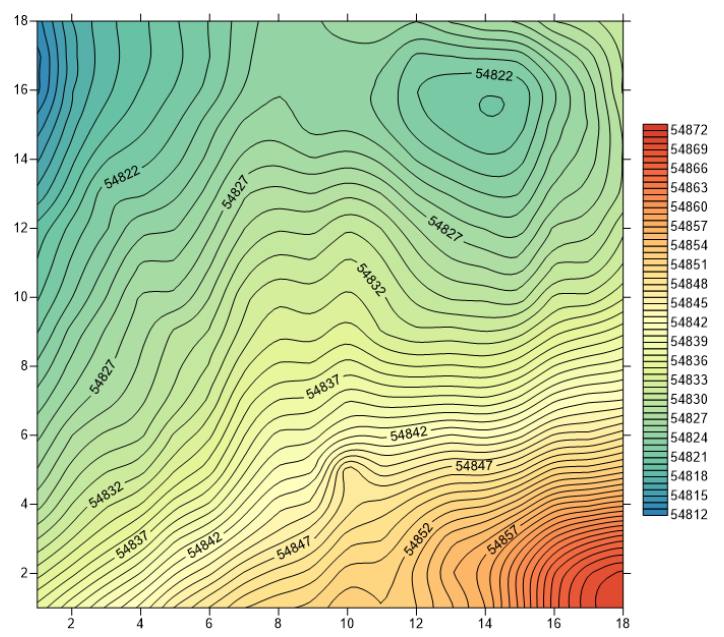

Figure 5. The Local Geomagnetic Contour Chart without Robot

In order to validate the local geomagnetic contour chart which was draw though this method matched the actual situation or not, the geomagnetic contour should be drawn in this region with no magnetic object. Therefore, the geomagnetic filed of this region was measured again when the magnetic object is moved. Then the geomagnetic contour in this region with no magnetic object is shown in Figure 5.

It can be seen when compared Figure 4 to Figure 5 that the trend of geomagnetic field in most part of region is same and the value at the same points in this region is little difference. The maximum deviation at the same points is only $2.2 \mathrm{nT}$. Compared to the local geomagnetic contour chart only using theoretical model data, the contour chart to be draw using this elimination method is more consistent with the actual situation. This method is better than theoretical model to deal with magnetic anomaly.

\section{Conclusion}

In the measurement of geomagnetic field, some measured data may be not accurate due to the presence of movable magnetic objects (such as cars, boats, etc.) in the measurement region. The precision of chart draw by all measured data is not high. In this paper, the influence on magnetic objects to geomagnetic field is analyzed, the method which can be improved the precision of geomagnetic chart is proposed. In this method, affected regions by magnetic object are gotten, the geomagnetic model are built in these affected region by precise data, the measured data in these region is replayed with model data, finally, the geomagnetic chart is draw by combined data. From the experimental results, the geomagnetic chart draw though this method is more consistent with the actual situation. Thus, this method can improve the accuracy of the entire region of the geomagnetic chart. 


\section{References}

[1] R. A. Langel, "International geomagnetic reference field: the sixth generation", Geomag. Geoelectr, vol. 44, (1992), pp. 679-708.

[2] IAGA Division V, "Working group 8.International geomagnetic reference field 1995 revision", Geomag. Geoeletr, vol. 47, (1996), pp. 1257-1261.

[3] IAGA Division V, "Working Group 8-International geomagnetic reference field 2000.Phys.Earth Planet. Inter.", vol. 120, (2000), pp. 39-42.

[4] T. J. Sabaka, N.Olsen and M. E. Purucker, "Extending comprehensive models of the Earth's magnetic field with Ørsted and CHAMP data”, Geophysical Journal International, vol. 195, no. 2, (2004), pp. 521-535.

[5] S. Maus1, M. Rother, K. Hemant, C.Stolle1, H. Lühr, A. Kuvshinov and N. Olsen, "Earth's lithospheric magnetic field determined to spherical harmonic degree 90 from CHAMP satellite measurements", Geophysical Journal International, vol. 164, no. 2, (2006), pp. 319-328.

[6] N. Olsen1, H.Lühr, T. J. Sabaka and M. Mandea, "CHAOS - a model of the Earth's magnetic field derived from CHAMP, Ørsted, and SAC-C magnetic satellite data", vol. 166, no. 4, (2006), pp. 67-75.

[7] Z. C. An, "Spherical cap harmonic analysis of geomagnetic field for China", Chinese J. Geophys, vol. 36, no. 6, (1993), pp. 753-764.

[8] Z. C. An, D. H. Tan and V. P. Golovkov, "Legendre polynomial models of the geomagnetic field over the eastern Asia at epoch 1980.0”, Chinese J. Geophys, vol. 38, no. 2, (1995), pp. 227-233.

[9] Z.-C. An and N. M. Rotanova, "Calculations and analyses of the geomagnetic field models for east Asia", Chinese J. Geophys, vol. 45, no. 1, (2002), pp. 34-41.

[10] P. C. Chen, "A detailed geomagnetic survey of Pehpei District", Szechuan, China.Chinese J.Geophys. (Acta Geophysica Sinica), vol. 1, no. 2, (1948), pp.177-186.

[11]Z. W. Gu, Z. C. An and J. T. Gao, "Spherical cap harmonic analysis on the geomagnetic field in BeijingTianjin-Hebei region”, Chinese J. Geophys, vol. 47, no. 6, (2004), pp. 61-66.

[12] J. -T. Gao, Z. -C. An, Z. -W. Gu and H. We, "Selection of the geomagnetic normal field and calculation of the geomagnetic anomalous field”, Chinese J. Geophys, vol. 47, no. 6, (2004), pp. 61-66. 
International Journal of Control Automation Vol. 7, No. 6, (2014) 